\title{
Letter to the Editor: Picolinic Acid as a Zinc- Binding Ligand in Human Milk: an Unconvincing Case
}

\author{
LUCILLE S. HURLEY AND BO LÖNNERDAL \\ Department of Nutrition, University of California, Davis, California USA
}

In the paper entitled "Characterization and Quantitation of a Zinc-Binding Ligand in Human Milk," Evans and Johnson (4) claim to have identified as picolinic acid a low-molecular-weight ligand binding zinc in human milk. This claim is in conflict with our report identifying this ligand as citrate (13). We now present some experimental data which we hope will clarify the issue. We also question some of the techniques, as well as conclusions, described in the paper by Evans and Johnson.

To evaluate the possible presence of picolinic acid in human milk, we subjected to gel filtration samples of (1) human milk, (2) picolinic acid plus zinc, and (3) human milk plus picolinic acid plus zinc. A column (1.6 x $90 \mathrm{~cm})$ was packed with Sephadex G15 (Pharmacia Fine Chemicals, Piscataway, NJ) that had been treated with $\mathrm{NaBH}_{4}$ to remove surface charges originally present on the gel matrix (11). Under these conditions, recovery of zinc from the column was 95 to $100 \%$; in contrast, when untreated gels are used, recovery of zinc is variable and often lower than $60 \%$ (3, 12). The column was equilibrated with $0.1 \mathrm{M}$ ammonium acetate buffer ( $\mathrm{pH} \mathrm{6.5)}$ at a flow rate of $60 \mathrm{ml} / \mathrm{hr}$. The fractions were analyzed for zinc by flame atomic absorption spectrophotometry (Perkin-Elmer 370) and for aromatic absorption at $280 \mathrm{~nm}$ (Varian, Cary 219).

As shown in Figure $1 A$, zinc in human milk eluted in two peaks. The first peak consisted of high-molecular-weight zinc-binding protein(s), and the second was a low-molecular-weight peak coeluting with citrate. This was demonstrated by a specific enzymatic assay for citrate (2), confirming our previous report (13).

Figure $1 B$ shows the elution behavior of picolinic acid plus zinc $[300 \mu \mathrm{M}$ picolinic acid $+0.3 \mu \mathrm{g} / \mathrm{ml}$ of zinc in the form of $\mathrm{Zn}$ $\left(\mathrm{NO}_{3}\right)_{2}$ ]. It is clear that picolinic acid coelutes with zinc, but at a position considerably different from that of the low-molecularweight zinc complex of human milk seen in Figure $1 A$. No such zinc peak was observed in the elution pattern of human milk. It can also be noted that this peak is positioned after the total volume of the column. This is in accordance with the elution behavior of aromatic compounds on Sephadex gels (8); such compounds are retarded because of hydrophobic interaction and thus elute after the total volume of the column.

When picolinic acid plus zinc (in the same concentrations as above) was added to human milk and this combination was subjected to gel filtration, three zinc-binding peaks were observed (Figure $1 C$ ). The first two peaks were the same as those shown in Figure $1 A$ for human milk; the third peak appeared in the same elution position as that shown for picolinic acid plus zinc in Figure $1 B$. Therefore, it is evident that if there were a zinc picolinate complex in human milk, this complex would elute after the total volume of the column. Our results demonstrate that zinc picolinate cannot be a significant naturally occurring zinc complex in human milk. Furthermore, examination of Figure 1 in Evans and Johnson's paper shows that their low-molecular-weight zinc complex eluted before the total volume of the column. This result is, in fact, in accordance with the elution pattern we observed for zinc citrate.
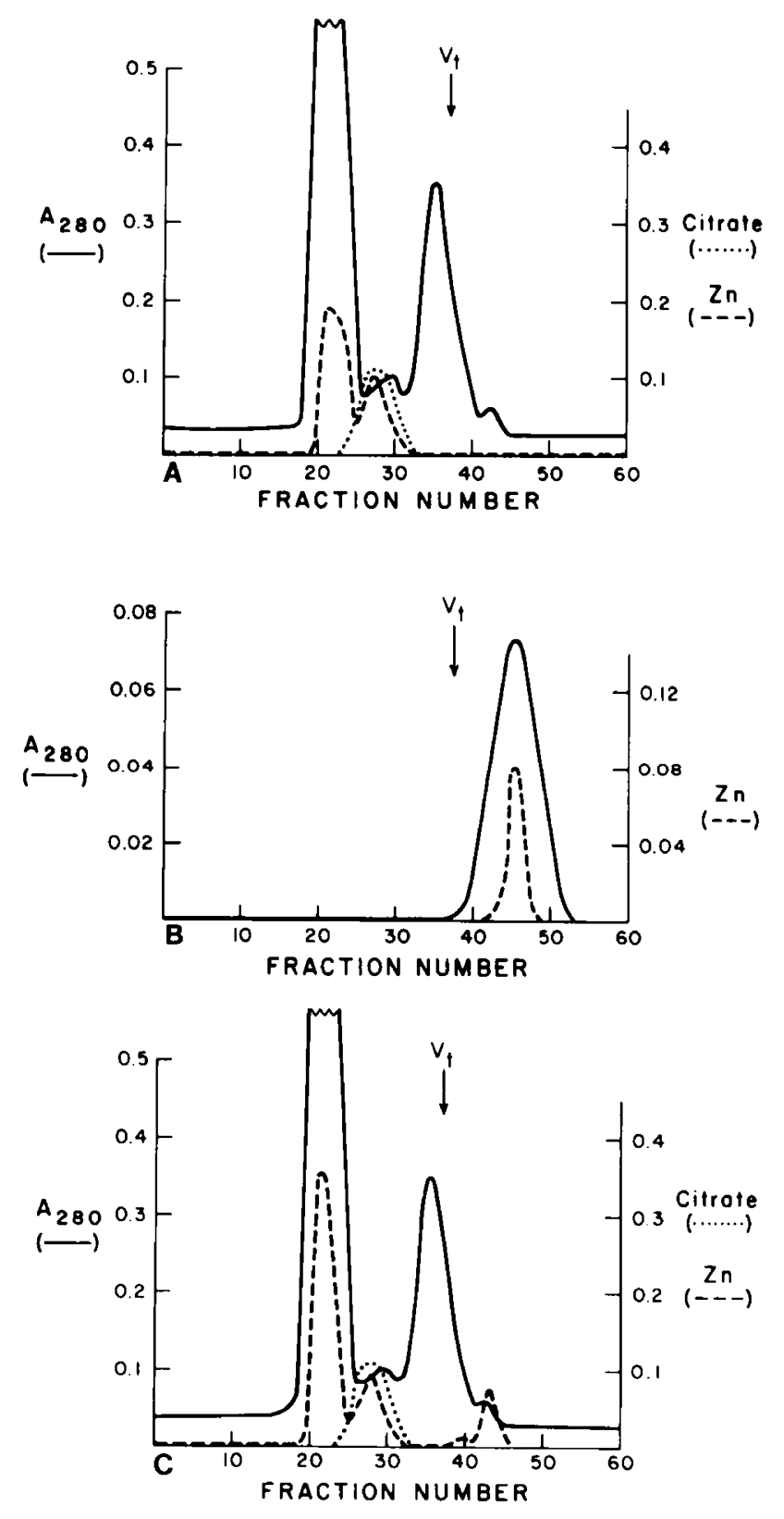

Fig. 1. Gel filtration chromatography on Sephadex G-15. Column size: $0.9 \times 90 \mathrm{~cm}$. Buffer: $0.1 \mathrm{M}$ ammonium acetate $\mathrm{pH} 6.5$. Flow rate: $60 \mathrm{ml}$ / hr. Samples: $A$, human milk; $B$, picolinic acid plus zinc; $C$, human milk plus picolinic acid plus zinc. 
We recognize that our method of gel filtration is different from that used by Evans and Johnson with respect to the amount of zinc in the buffers, as well as the charges on the gel. However, these differences would not affect the relative positions of the peaks in the chromatograms. Therefore, the discrepancy between the results we present here and the conclusions of Evans and Johnson cannot be explained by a difference in methodology. It should also be pointed out that Evans and Johnson have not shown simultaneous elution curves for zinc and picolinic acid in human milk.

Another questionable aspect of this paper concerns the method used for "quantitation" of picolinic acid in human milk. Evans and Johnson took one pooled sample of human milk, subjected it to ultrafiltration, gel filtration, and ion-exchange chromatography, and then measured the absorption at $265 \mathrm{~nm}$ of the low-molecularweight zinc peak. This procedure provides a very nonspecific measure of picolinic acid, as all compounds with an aromatic structure absorb at this wave length. In fact, all the aromatic metabolites of tryptophan, including quinolinic acid and 3-hydroxyanthranilic acid, exhibit similar absorption spectra. It is likely that the errors inherent in this procedure, as well as methodologic problems, have led Evans and Johnson to a calculation of the concentration of picolinic acid in human milk that seems unreasonable in light of other known information about tryptophan and its metabolites in biologic fluids.

Although our results show that the low-molecular-weight zinc complex normally found in human milk is not zinc picolinate, it might be considered theoretically possible for such a compound to be present. However, the concentration of free tryptophan in human milk is very low, and picolinic acid is known to be a minor metabolite of this amino acid $(6,14)$. In fact, it has been shown that only $7.4 \%$ of 3-hydroxyanthranilic acid, an intermediate metabolite of tryptophan, was converted to picolinate by rat liver extracts (14). The value given by Evans and Johnson for picolinic acid in human milk $(38 \mu \mathrm{g} / \mathrm{ml})$ greatly exceeds the concentration of free tryptophan in human milk $[<3 \mu \mathrm{g} / \mathrm{ml}(15)]$. Their value for picolinic acid is also considerably higher (by a factor of 22) than that reported for the concentration of nicotinic acid (another metabolite of tryptophan) in human milk $(1.7 \mu \mathrm{g} / \mathrm{ml})(1)$. However, according to the data presented by Evans and Johnson, they actually found considerably less picolinic acid than nicotinic acid in human milk (Ref. 4, Fig. 2B). Thus, their own data are inconsistent with their statement that human milk contains $38 \mu \mathrm{g} /$ $\mathrm{ml}$ of picolinic acid. Their conclusion that "the probability for the formation of a zinc:picolinic acid complex is high in human milk as well as in the intestinal lumen of infants consuming human milk" therefore seems unwarranted.

Part of the argument of Evans and Johnson is based on experimental data demonstrating improvement of zinc status in rats fed picolinic acid. We agree that picolinic acid is a good chelator, and it is well known that chelating agents may improve zinc absorp- tion. Similarly, other chelators such as EDTA $(5,9)$ and diiodohydroxyquinoline (7) (which are not normal metabolic compounds) have been shown to improve zinc absorption. However. the ability of a substance to improve zinc absorption does not mean that it plays a normal physiologic role in this process.

It must be noted that, based on the identification by Evans and Johnson of picolinic acid as a low-molecular-weight zinc binding ligand in human milk, zinc picolinate has been fed to an infant in an effort to improve its zinc status (10). The data and the arguments we have presented here suggest that Evans and Johnson's identification of picolinic acid and its role in zinc absorption is incorrect. The use of zinc picolinate in children must, therefore, in our opinion be questioned and carefully evaluated, particularly because picolinic acid is a known irritant (16).

\section{REFERENCES AND NOTES}

1. Blaxter, K. L.: Lactation and the growth of the young. In: S. K. Con, A. T. Cowie: Milk: The Mammary Gland and Its Secretion. Vol. II, p. 349 (Academic Press, Inc., New York, 1961)

2. Dagley, S.: Citrate UV spectrophotometric determination. In: H. O. Bergmeyer: Methods of Enzymatic Analysis. Vol. 3, p. 1562 (Academic Press, Inc., New York, 1974).

3. Evans, G. W., Grace, C. I., and Votava, H. J.: A proposed mechanism for zinc absorption in the rat. Am. J. Physiol., 228: 501 (1975).

4. Evans, G. W., and Johnson, P. E.: Characterization and quantitation of a zincbinding ligand in human milk. Pediatr. Res., 14: 876 (1980)

5. Forbes, R. M.: Excretory patterns and bone deposition of zinc, calcium and magnesium in the rat as influenced by zinc deficiency, EDTA and lactose. J. Nutr., 74: 194 (1961).

6. Henderson, L. M., and Swan, P. B.: Picolinic acid carboxylase. Methods Enzymol., 18B: 175 (1971).

7. Jackson. M. J.: Zinc and di-iodohydroxyquinoline therapy in acrodermatitis enteropathica. J. Clin. Pathol., 30: 284 (1977).

8. Janson, J.-C.: Adsorption phenomena on Sephadex. J. Chromatogr., 28: 12 (1967).

9. Kratzer, F. H., Allred, J. B., Davis, P. N., Marshall, B. J., and Vohra, P.: The effect of autoclaving soybean protein and the addition of ethylenediaminetetraacetic acid on the biological availability of dietary zinc for turkey poults. J. Nutr., 68: 313 (1959)

10. Krieger, I.: Picolinic acid in the treatment of disorders requiring zinc supplementation. Nutr. Rev., 38: 148 (1980)

11. Lönnerdal, B.: Chemical modification of dextran gels for gel filtration of trace element ligands. In: P. Schramel: Trace Element Analytical Chemistry in Medicine and Biology. Proceedings of the First International Workshop (in press).

12. Lönnerdal. B.. Keen, C. L.. Sloan, M. V., and Hurley, L. S.: Molecular localization of zinc in rat milk and neonatal intestine. J. Nutr., 110: 2416 (1980).

13. Lönnerdal, B., Stanislowski, A. G., and Hurley, L. S.: Isolation of a low molecular weight zinc binding ligand from human milk. J. Inorg. Biochem., 12: 71 (1980).

14. Suhadolink, R. J., Stevens, C. O., Decker, R. H., Henderson, L. M., and Hankes, L. V.: Species variation in the metabolism of 3-hydroxyanthranilate for pyridinecarboxylic acids. J. Biol. Chem., 228: 973 (1957).

15. Wurtman, J. J., and Fernstrom, J. D.: Free amino acid, protein, and fat contents of breast milk from Guatemalan mothers consuming a corn-based diet. Early Hum. Dev., 3: 67 (1979)

16. Merck Co.

17. This research was supported in part by NIH Research Grant HD-12547 from the National Institute of Child Health and Human Development. 